\title{
A NOTE ON FINITELY GENERATED GROUPS
}

\author{
ROBERT J. GREGORAC
}

We here generalize a result of Murasugi for finitely presented groups ([2]; see Corollary 3 below) to arbitrary finitely generated groups.

We begin with a simple extension of a well-known result for abelian groups.

THEOREM 1. Let $G=H L$, where $H$ is a subgroup of $G$ and $L$ is a subgroup of the center of $G$. If $G / H$ is a direct product, $G / H=T_{1} \times K$, where $K$ is a free abelian group, then $G$ is a direct product, $G=T \times C$, where $H \triangleleft T, T / H \cong T_{1}, C \triangleleft L$ and $C \cong K$.

In particular, if $T_{1}$ is finite then the index of $H$ in $T$ is finite.

Proof. Since $L$ is in the center of $G, H$ is normal in $H L=G$. Let $T$ be the preimage of $T_{1}$ under the natural homomorphism $G \rightarrow G / H$. For each element $k$ of a fixed basis of $K$ choose an element $c \in L$ which maps on to $k$ and let $C$ be the group generated by these elements $c$ of $L$. The group $C$ is in the center of $G$, so $C \triangleleft G$ and if $x \in C \cap T$, then $x=c_{1}{ }^{r_{1}} C_{2}{ }^{r_{2}} \cdots c_{n}{ }^{{ }}$, so $x$ maps on to $k_{1}{ }^{{ }_{1}} \cdots k_{n}{ }^{{ }}{ }_{n} \in T_{1} \cap K=1$. Since $K$ is free abelian $r_{1}=r_{2}=\cdots=r_{n}=0$, and thus $C \cap T=1$. Hence $G$ is the direct product $T \times C$. Finally, $H \subseteq T$, so $C \cap H=1$ and $C \cong C / C$ $\cap H \cong C H / H=K$, completing the proof.

Corollary 1. Let $G=H L$, where $H$ is a subgroup of $G$ and $L$ is a subgroup of the center of $G$. If $G / H$ is finitely generated, then $G$ is a direct product $G=T \times C$, where $H \triangleleft T, C \triangleleft L$ and the index of $H$ in $T$ is finite.

Proof. The group $G / H$ is the direct product of a finite group $T_{1}$ and a free abelian group $K$. The corollary now follows from the theorem.

Corollary 2. Let $H$ be a subgroup of the finitely generated group $A$ and let $L$ be an abelian subgroup of $C_{A}(H)$, the centralizer of $H$ in $A$. If $H L$ is of finite index in $A$, then $H$ is finitely generated.

Proof. The group $G=H L$ is finitely generated, because it has finite index in the finitely generated group $A[3,8.4 .33]$. As a homomorphic image of $G, G / H$ must be finitely generated, so by corollary 1 , $G=T \times C$ and $H$ has finite index in $T$. But $T$ is a homomorphic image of $G$, so $T$ is finitely generated and hence $H$ is finitely generated also.

Received by the editors June 27, 1966 and, in revised form, August 1, 1966. 
In particular, note that if $Z$ is the center of the finitely generated group $A$ and $H Z$ has finite index in $A$, then $H$ is finitely generated.

Corollary 3. Let $A$ be a finitely generated group and let $H$ be a normal subgroup of $A$ such that $A / H$ is infinite cyclic. If the centralizer of $H$ in $A$ is not contained in $H$, then $H$ is finitely generated.

Proof. If $C_{A}(H) \nsubseteq H$, let $x \in C_{A}(H), x \in H$ and let $L=\langle x\rangle$. Then $H L / H$ is a nontrivial subgroup of the integers $A / H$ and has finite index, so $H L$ has finite index in $A$. The result now follows from Corollary 2.

Corollary 4. Let $A, H$ and $L$ satisfy the assumptions of Corollary 2. Then (i) $A$ is residually finite if and only if $H$ is residually finite and (ii) A satisfies the maximal condition for subgroups if and only if $H$ satisfies the maximal condition.

Proof. In the proof of corollary 2 we noted that $G=H L=T \times C$, where $H \triangleleft T$. If $H$ is residually finite [3], then so is $T$, because it is a finite extension of $H$. Then $T \times C$ is residually finite, because the direct sum of residually finite groups is residually finite. Now $[G: H L]$ is finite so there is a normal subgroup $N$ of $G$ having finite index in $G$ and contained in $H L$. Hence $G$ is residually finite because $N$ is residually finite as a subgroup of $H L$ and a finite extension of a residually finite group is residually finite.

If $H$ satisfies the maximal condition, so does $H L$ because $H L / H$ satisfies the maximal condition.

As before, there is a normal subgroup $N$ of $G$ of finite index in $G$ which must satisfy the maximal condition, because it is a subgroup of $H L$. Hence $G$ also satisfies the maximal condition.

The other statements are clear.

A group $G$ is called an $F C$ group if and only if every element of $G$ has only a finite number of conjugates.

Corollary 5. Let $A$ be a finitely generated group with center $Z$. If $H$ is a periodic $F C$ group such that $H Z$ has finite index in $A$, then $A$ is an FC group.

In particular, $[A: Z]$ and $\left[A^{\prime}: 1\right]$ are finite.

Proof. (See [3, Chapter 15] for the material on $F C$ groups used here.)

By the remark after Corollary 2, $H$ is finitely generated, and a periodic $F C$ group is locally normal, so $H$ is a finite group. Note that $H Z / Z \cong H / H \cap Z$ is finite, so $[A: Z]=[A: H Z][H Z: Z]$ is finite. Hence $A$ is an $F C$ group. 
Let $\phi(G)$ denote the Frattini subgroup of $G$.

Corollary 6. Let $A$ be a finitely generated group with center $Z$. Then (i) $A$ is abelian if and only if $A=\phi(A) Z$ and (ii) $A$ is an FC group if and only if $\phi(A) Z$ has finite index in $A$ and $\phi(A)$ has an abelian subgroup $S$ of finite index.

Proof. If $A=\phi(A) Z$, then by Corollary $2, \phi(A)$ is finitely generated. Since $\phi(A)$ is the set of nongenerators of $A$ we have that $\phi(A) Z=Z$, so $A=Z$ is abelian. The converse is clear.

If $\phi(A) Z$ has finite index in $A$, then the abelian group $S Z$ has finite index in $A$, so $\phi(A)$ is finite and nilpotent by a result of $\mathrm{P}$. Hall $[1$, Lemma 10] and hence $[A: Z]$ is finite. Thus $A$ is an $F C$ group.

Conversely, if $A$ is an $F C$ group, then $[A: Z]$ is finite and by Hall's result $\phi(A)$ is finite and nilpotent, so $\phi(A)$ has an abelian subgroup of finite index.

Added In Proof. We note that the idea in Theorem 1 can be extended, so that theorem 1 is a special case of the following

Theorem A. Let $V(L)$ be the variety of groups satisfying the identical relations $L$.

If $G=H B, B \in V(L), H \triangleleft G$, and $G / H$ maps homomorphically onto a free group $K$ in the variety $V(L)$, then $G$ splits over $K$, that is, $G=T C$, $T \triangleleft G, C \cong K$ and $T \cap C=1$.

Proof. Since $K \in V(L), K \cong F / N$, where $F$ is an ordinary free group on the set $\left\{f_{i} \mid i \in I\right\}$ and $N$ is a fully invariant subgroup of $F$ determined by the laws $L$.

Let $k_{i}=f_{i} N, i \in I$ and let $T$ be the kernel of the homomorphism $\theta$ from $G$ onto $K$. We may assume that $k_{i} \neq 1, i \in I$. Choose elements $c_{i}$ in $B$ but not $T$ such that $c_{i}$ maps onto $k_{i}, i \in I$. Let $C$ be the subgroup of $G$ generated by the $c_{i}$. Let $w=\prod_{m=1}^{q} c_{i_{m}}^{e_{m}}, e_{m}= \pm 1, i_{m} \in I$ be an element $T \cap C$. Then $w \theta=\prod_{m=1}^{a} k_{i_{m}}^{e_{m}}=1$ in $K$, so $\prod_{m=1}^{q} x_{i_{m}}^{e_{m}}=1$ is an identical relation on any group of the variety $V(L)$. Now $C \subseteq B$, so $C \in V(L)$, and we have that $w=1$, so $T \cap C=1$. Finally, $K \cong G / T$ $=C T / T \cong C / C \cap T \cong C$.

\section{REFERENCES}

1. P. Hall, On the finiteness of certain soluble groups, Proc. London Math. Soc. (3) 9 (1959), 595-622.

2. Kunio Murasugi, On the center of the group of a link, Proc. Amer. Math. Soc. 16 (1965), 1052.

3. W. R. Scott, Group theory, Prentice-Hall, Englewood Cliffs, N. J., 1964.

Iowa State University 